\title{
Switching dynamics of dark-pulse Kerr frequency comb states in optical microresonators
}

\author{
Elham Nazemosadat $\odot,{ }^{1, *}$ Attila Fülöp, ${ }^{1, \dagger}$ Óskar B. Helgason, ${ }^{1}$ Pei-Hsun Wang, ${ }^{2, \$}$ Yi Xuan, ${ }^{3,}{ }^{\S}$ Dan E. Leaird, ${ }^{2}$ \\ Minghao Qi ${ }^{2,3}$ Enrique Silvestre $\odot,{ }^{4}$ Andrew M. Weiner $\odot,{ }^{2,3}$ and Victor Torres-Company ${ }^{1, \|}$ \\ ${ }^{1}$ Department of Microtechnology and Nanoscience (MC2), Chalmers University of Technology, SE-41296 Göteborg, Sweden \\ ${ }^{2}$ School of Electrical and Computer Engineering, Purdue University, West Lafayette, Indiana 47907-2035, USA \\ ${ }^{3}$ Birck Nanotechnology Center, Purdue University, West Lafayette, Indiana 47907-2035, USA \\ ${ }^{4}$ Department of Optics-ICMUV, University of Valencia, 46100 Burjassot, Valencia, Spain
}

(Received 23 November 2019; revised 19 October 2020; accepted 21 December 2020; published 14 January 2021)

\begin{abstract}
Dissipative Kerr solitons are localized structures that exist in nonlinear optical cavities. They lead to the formation of microcombs - chip-scale frequency combs that could facilitate precision frequency synthesis and metrology by capitalizing on advances in silicon photonics. Previous demonstrations have mainly focused on anomalous dispersion cavities. Notwithstanding, localized structures also exist in the normal dispersion regime in the form of circulating dark pulses, but their physical dynamics is far from being understood. Here, we explore dark-pulse Kerr combs generated in normal dispersion optical microresonators and report the discovery of reversible switching between coherent dark-pulse combs, whereby distinct states can be accessed deterministically. Furthermore, we reveal that the formation of dark-pulse Kerr combs is associated with the appearance of another resonance, a feature that has never been observed for dark pulses and is ascribed to soliton behavior. These results contribute to understanding the nonlinear physics in normal dispersion nonlinear cavities and provide insight into the generation of microcombs with high conversion efficiency.
\end{abstract}

DOI: 10.1103/PhysRevA.103.013513

\section{INTRODUCTION}

Dissipative solitons are self-enforcing, stationary structures that exist in diverse nonlinear dissipative systems subject to an external pump of energy [1]. The recent discovery of temporal dissipative solitons in optical cavities displaying Kerr nonlinearity [2,3] (from now on dissipative Kerr solitons or DKS) has facilitated the investigation of their rich dynamics [4-15]. DKS rely on balancing the inherent cavity dispersion with the corresponding Kerr nonlinear phase shift induced by the soliton, while the dissipative nature of the cavity is offset by supplying it with the energy from a pump laser. DKS are just one particular solution of the complex spatiotemporal landscape in nonlinear Kerr cavities [5,6]. The same optical cavities can also display chaos, breathing dynamics [7-10], soliton crystals [11,12], and transitions between some of these

\footnotetext{
*Present address: ITEAM Research Institute, Universitat Politécnica de Valéncia, 46022 Valencia, Spain.

${ }^{\dagger}$ Present address: OptiGOT AB, SE-41133 Göteborg, Sweden.

¥Present address: Department of Optics and Photonics, National Central University, Taoyuan City 32001, Taiwan.

${ }^{\S}$ Present address: Department of Surgery, Indiana Center for Regenerative Medicine and Engineering, Indiana University School of Medicine, Indianapolis, Indiana 46202, USA.

"torresv@chalmers.se
}

Published by the American Physical Society under the terms of the Creative Commons Attribution 4.0 International license. Further distribution of this work must maintain attribution to the author(s) and the published article's title, journal citation, and DOI. Funded by Bibsam. states [13]. The single-soliton regime can be accessed deterministically by decreasing the number of cavity solitons, while properly tuning the pump laser over the resonance [14]. Mapping this complexity is not only of fundamental interest, but important for the design and operation of stable, ultrabroadband coherent Kerr combs $[4,15]$, which have potential applications in multiple fields, ranging from optical clocks to coherent communications [16-26].

DKS require the optical cavity to display anomalous dispersion [27] at the pump wavelength. Interestingly, other stationary structures such as ultrashort optical pulses [28] or dark-pulse Kerr combs [29] can be found in cavities operating in the normal dispersion regime [i.e., decreasing free spectral range (FSR) with optical frequency]. The time-domain waveform of a dark-pulse Kerr comb corresponds to a localized dark-pulse structure, where low-intensity oscillations are embedded in a high-intensity background. These pulses can be interpreted as two stably interlocked switching waves, connecting the upper and lower homogeneous steady-state solutions of the bistability curve in Kerr cavities [30]. These localized waveforms also exhibit breathing dynamics [31,32] and have intriguing connections to sneaker waves found in hydrodynamics, called flaticons [33] and platicons [34] in optics. In comparison to DKS, the physics of dark-pulse Kerr combs is less understood, even though these combs are more efficient in converting the pump power into useful comb light [35] —an aspect that is particularly promising for coherent optical communications [36,37]. Some key questions remain unanswered, such as what the pathway to their generation is, starting from a continuous-wave (CW) waveform, and whether this transition is accompanied by similar switching dynamics to what has been observed in DKS. 
(a)

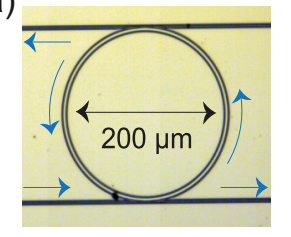

(b)

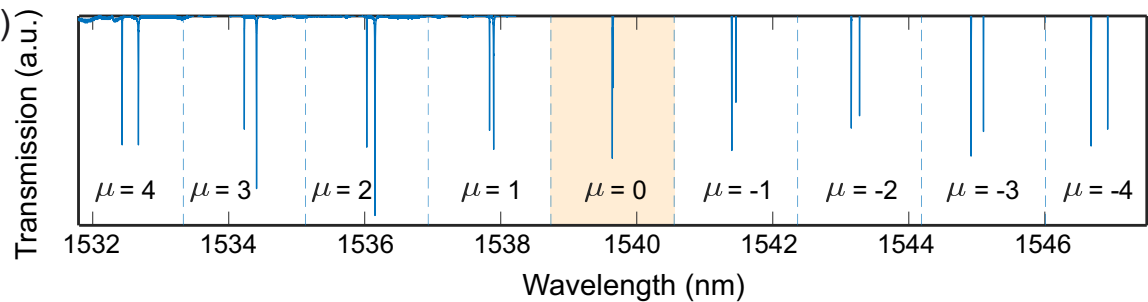

(c)

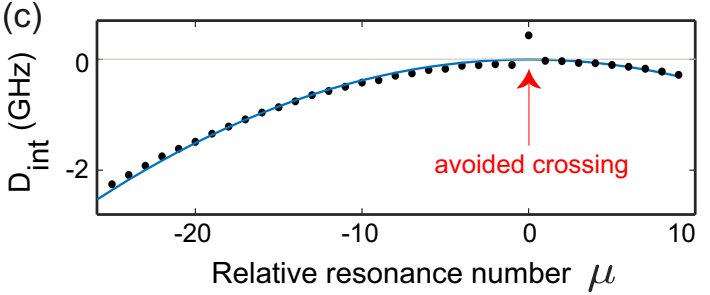

(d)

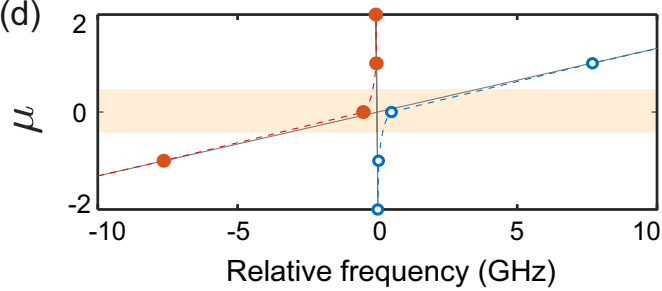

FIG. 1. Linear characterization of the multimode silicon nitride microresonator. (a) Microscope image of the silicon nitride microresonator. (b) Measured transmission scan of the microresonator, where two clear transverse modes appear. (c) Integrated dispersion, i.e., frequency deviation of the resonance locations of the main mode (black dots), $\omega_{\mu}$, with respect to an ideal grid, $D_{\text {int }}=\left(\omega_{\mu}-\omega_{0}-2 \pi D_{1} \mu\right) / 2 \pi$, where $\mu$ is the mode number and $D_{1}$ the free spectral range. The main mode displays normal dispersion, while at $\mu=0$ the dispersion changes locally to anomalous due to the linear coupling between two transverse modes in the resonator. (d) Linear mode coupling effect. The transmission spectrum is divided into blocks with a spacing difference of 1 FSR [dotted lines in (b)] to plot this diagram and calculate the linear coupling strength. The red and blue circles represent the resonance frequencies of the two transverse modes in different FSR blocks. The gray solid lines depict the no mode coupling $(\kappa=0)$ case, found by linearly fitting $\mu$ versus the resonance frequencies in the region far from $\mu=0$. Mode coupling shifts the resonances apart from each other at $\mu=0$, leading to an avoided mode crossing. The measured linear coupling coefficient between the modes is $\kappa=22.7 \mathrm{~m}^{-1}$, while the measured group velocity dispersion of the main mode is $\beta_{2}=139 \mathrm{ps}^{2} / \mathrm{km}$.

In this work, without loss of generality, normal dispersion optical microresonators are used to explore the physical dynamics of dark-pulse Kerr combs. We report deterministic switching between dark-pulse Kerr comb states and support our results with numerical simulations that take into account the linear coupling between the dominant transverse modes of the microresonator. The numerical analysis shows that each comb state is uniquely ascribed to a number of low-intensity oscillation periods. This number can be deterministically controlled and increased or decreased one at a time, unraveling an overlooked dependence with the pump laser detuning parameter for dark-pulse Kerr combs. This tuning of the dark-pulse duration and shape is continuous and not associated with sharp changes, which is attributed to the interaction between the transverse modes. Strikingly, we find that the formation of dark-pulse Kerr combs is also accompanied by the appearance of an extra resonance, in compelling similarity to the behavior reported for DKS [14] and perfect soliton crystals [13]. In contrast, however, our measurements reveal that in dark-pulse Kerr combs, the pump is effectively blue detuned with respect to the cavity resonance that is Kerr shifted due to the highpower CW background of the dark pulse. The appearance of an extra resonance is not dependent on the linear coupling between the transverse modes. While the observations reported here have been made in a normal dispersion microresonator, similar results might be found in nonlinear systems with selffocusing nonlinearity and normal dispersion, such as fiber ring cavities, Bose-Einstein condensates, or hydrodynamics $[33,38,39]$.

\section{MICRORESONATOR CHARACTERIZATION}

A silicon nitride microresonator with a designed cross section of $2 \mu \mathrm{m}$ width $\times 600 \mathrm{~nm}$ height is used in our experiments. The ring features a radius of $100 \mu \mathrm{m}$ [Fig. 1(a)], corresponding to an FSR of around $229 \mathrm{GHz}$ for the main mode used for comb generation, with a measured mean intrinsic $Q$ factor of around $1.6 \times 10^{6}$. The particular modes of interest are $\mathrm{TE}_{1}$ and $\mathrm{TE}_{2}$, which exhibit normal dispersion within the $\mathrm{C}$ band [40].

A tunable external-cavity pump laser with sub-10 kHz linewidth is calibrated using a Mach-Zehnder interferometer [28], and scanned over the $\mathrm{C}$ band to find the resonance locations of the two linearly coupled transverse modes. The measured transmission scan is shown in Fig. 1(b). As observed, around $1540 \mathrm{~nm}$, the resonance frequencies of the two transverse modes are very close to each other. Thus, any fabrication imperfections could lead to linear coupling between the modes [41]. Mode coupling, in turn, induces a frequency shift on the interacting resonances and pushes them apart, leading to an avoided mode crossing [Fig. 1(d)] [42,43]. This effect locally modifies the dispersion of the modes and results in a local anomalous dispersion [Fig. 1(c)], where it is possible to achieve modulational instability and initialize the dark-pulse Kerr comb $[29,44]$.

For comb generation, the pump power is amplified in an erbium-doped fiber amplifier and optically filtered to remove the amplified spontaneous emission noise far away from the pump [Fig. 2(a)]. This increases the signal quality of the generated comb lines. The microresonator chip is placed on a 


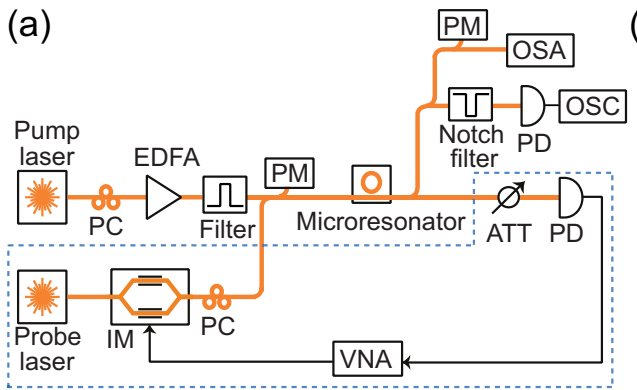

(d)
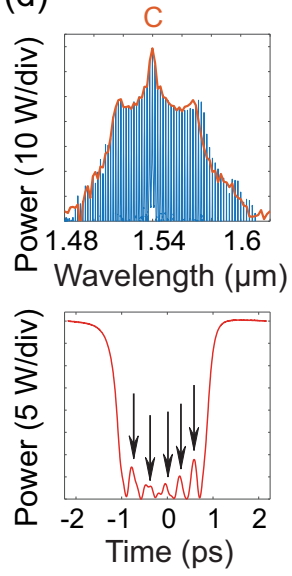

(b)

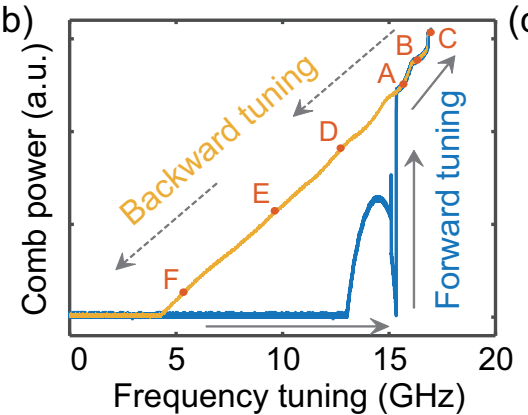

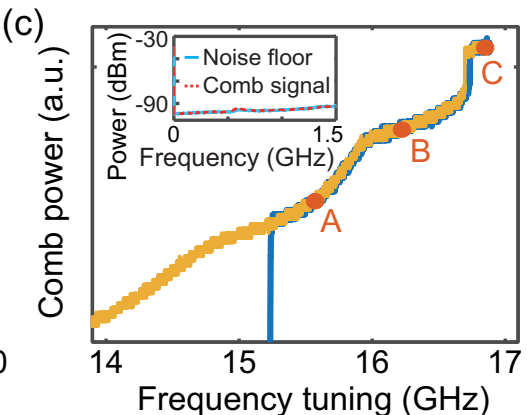

FIG. 2. Deterministic switching of dark pulses. (a) Setup for comb generation and measurement. The setup within the dashed lines is used in Sec. IV. PC: Polarization controller, EDFA: Erbium-doped fiber amplifier; PM: Power meter; ATT: Attenuator; PD: Photodiode; IM: Intensity modulator; OSA: Optical spectrum analyzer; OSC: Oscilloscope; VNA: Vector network analyzer. (b) Measured comb power when the pump is forward and backward tuned, shown in blue (dark gray) and yellow (light gray), respectively. The frequency axis indicates the pump location with respect to its initial frequency. The frequency scan is calibrated by means of an auxiliary interferometer. (c) Zoomed-in view of (b), where smoothed steplike patterns are observed as the pump is tuned, indicating switching between dark-pulse comb states. The inset shows the radio-frequency spectrum (dashed red) and noise floor (solid blue) of the generated comb. (d) The blue frequency lines are the comb spectra measured at different pump detunings, corresponding to the comb states marked in (b). The comb envelope of each state is simulated and shown in red, with the corresponding simulated time-domain waveforms underneath. The arrows point the number of low intensity oscillations. For state F, the phase of the pulse is also shown in dashed blue.

piezo-controlled positioning stage, which is temperature controlled with a standard laser temperature controller at $18^{\circ} \mathrm{C}$, limiting the variations to less than $0.01{ }^{\circ} \mathrm{C}$. This allows stable comb operation over several hours. The pump is coupled into the microresonator using a lensed fiber. The off-chip power is $25.6 \mathrm{dBm}$. At high pump powers, the coupling losses between the fiber and chip are estimated to be $4-5 \mathrm{~dB}$ per facet. Our simulations, explained in Sec. III, have a good agreement with the experimental measurements when the coupled pump power is assumed $150 \mathrm{~mW}(21.8 \mathrm{dBm})$. The set of two hybridized resonances that experience the strongest linear coupling, indicated by the red arrow in Fig. 1(c), are pumped from the blue side. The same microresonator, pumped in the same way, has been previously used to generate a modelocked Kerr frequency comb, with evidence of dark pulses circulating in the cavity [36].

\section{SWITCHING DYNAMICS OF DARK-PULSE COMB STATES}

As the pump is tuned over the resonance from the thermally stable blue side towards the red $[3,45]$ (forward tuning), a coherent dark-pulse Kerr comb is generated. The comb state is monitored using an optical spectrum analyzer, while the power in the generated comb lines is measured with an oscilloscope, after suppressing the pump line with an optical notch filter [Fig. 2(a)]. As the laser is tuned further into resonance, an increased comb power is observed. The comb power increases in a gradual manner while displaying continuous steps, as shown in the topmost parts of Figs. 2(b), 2(c) (positions $\mathrm{A} \rightarrow \mathrm{B} \rightarrow \mathrm{C}$ ). Each step corresponds to a coherent comb state, indicated by a low-amplitude noise as shown in the inset of Fig. 2(c), which can be accessed sequentially. Keeping the pump power fixed, the comb power measurements in the forward pump tuning are repeated 100 times and smoothed steplike patterns, almost identical to those shown in Fig. 2(b), are measured. The dynamics reveal that at the used power level, the comb does not go over a chaotic state, making the comb generation process repeatable and deterministic. The comb found in state $\mathrm{A}$ achieves a conversion efficiency of around $25 \%$, where the conversion efficiency is defined as the output power in the comb lines (excluding the pump) divided by the input pump power. 
To get a better insight into the physics of comb generation in the normal dispersion regime, we simulate our experimental findings using an Ikeda map [46-48], modified such that the linear mode coupling is taken into account. Each round trip has two steps, one is the coupling between the pump in the bus waveguide and the ring and the other is the light propagation in the microresonator. The coupling between the pump to the modes in the resonator is found through coupled mode theory [49,50], assuming a $3 \times 3$ lossless coupler. It can be expressed as

$$
\left[\begin{array}{c}
A_{\text {out }} \\
A_{1}^{(k+1)} \\
A_{2}^{(k+1)}
\end{array}\right]=\left[\begin{array}{ccc}
\sqrt{1-\theta_{1}-\theta_{2}} & -\sqrt{\theta_{1}} & -\sqrt{\theta_{2}} \\
\sqrt{\theta_{1}} & \frac{\theta_{2}+\theta_{1} \sqrt{1-\theta_{1}-\theta_{2}}}{\theta_{1}+\theta_{2}} & \frac{\sqrt{\theta_{1} \theta_{2}}}{\theta_{1}+\theta_{2}}\left(\sqrt{1-\theta_{1}-\theta_{2}}-1\right) \\
\sqrt{\theta_{2}} & \frac{\sqrt{\theta_{1} \theta_{2}}}{\theta_{1}+\theta_{2}}\left(\sqrt{1-\theta_{1}-\theta_{2}}-1\right) & \frac{\theta_{1}+\theta_{2} \sqrt{1-\theta_{1}-\theta_{2}}}{\theta_{1}+\theta_{2}}
\end{array}\right]\left[\begin{array}{c}
A_{\text {in }} \\
A_{1}^{(k)} \\
A_{2}^{(k)}
\end{array}\right]
$$

where $A_{\text {in }}$ and $A_{\text {out }}$ are the input pump and throughput field of the microresonator. $A_{m}^{(k)}$ is the intracavity field of mode $m$ at round trip $k$ in the cavity and $\theta_{m}$ is the coupling coefficient between the bus and the microring for mode $m$. The evolution of the fields in the resonator is modeled using the nonlinear Schrödinger equation in multimode waveguides [51,52]. The propagation of mode $m$ in every round trip of the microresonator is given by

$$
\begin{aligned}
\frac{\partial A_{m}}{\partial z}= & \frac{-\alpha_{m}}{2} A_{m}+i \beta_{0}^{(m)} A_{m}-\beta_{1}^{(m)} \frac{\partial A_{m}}{\partial t}-i \frac{\beta_{2}^{(m)}}{2} \frac{\partial^{2} A_{m}}{\partial t^{2}} \\
& +i \gamma_{m}\left|A_{m}\right|^{2} A_{m}+i \kappa_{m n} A_{n \neq m},
\end{aligned}
$$

where $\alpha_{m}$ is the propagation loss, $\beta_{1}^{(m)}$ is the inverse group velocity, $\beta_{2}^{(m)}$ is the group velocity dispersion, and $\gamma_{m}$ is the nonlinear coefficient of mode $m$, while $\kappa_{m n}$ is the mode coupling strength between modes $m$ and $n$. The resonator length is $L$ and the linear phase shift of the field is $\beta_{0}^{(m)} L=-\delta_{0}^{(m)}$, where $\delta_{0}^{(m)}$ is the pump detuning from the cold-cavity resonance of each transverse mode closest to the pump frequency $\omega_{p}$. It can be expressed as $\delta_{0}^{(m)}=-\left[\beta^{(m)}\left(\omega_{p}\right)-\beta^{(m)}\left(\omega_{0, m}\right)\right] L$, where $\beta^{(m)}(\omega)$ is the propagation constant of mode $m$ at frequency $\omega$ and $\omega_{0, m}$ denotes the pumped cold-cavity resonance frequency of mode $m$. Note that nonlinear intermodal coupling [53] is not included in our simulations, assuming that it is negligible.

In each round trip a CW pump together with quantum noise consisting of one photon per spectral bin with random phase [47] is coupled to the ring. Unlike previously reported models that start the simulations with an initial intracavity square dark pulse, the initial intracavity field here is just the quantum noise in the resonator. The closest resonance to the avoided crossing is pumped from the blue side and the detuning is dynamically changed to emulate the tuning of the pump laser. The propagation in the ring is carried out using the split-step Fourier method. The parameters used in the simulations are extracted from the transmission scan measurements (Fig. 1). For the main mode, $\alpha_{1}$ corresponds to $0.1 \mathrm{~dB} / \mathrm{cm}, \theta_{1}=0.004$, the initial pump detuning is $\delta_{0}^{(1)}=-0.001 \mathrm{rad}, \beta_{2}^{(1)}=139 \mathrm{ps}^{2} / \mathrm{km}$, and $\mathrm{FSR}_{1}=229.08 \mathrm{GHz}$. For the auxiliary mode, $\alpha_{2}$ corresponds to $0.3 \mathrm{~dB} / \mathrm{cm}, \theta_{2}=0.01, \delta_{0}^{(2)}=-0.0033 \mathrm{rad}$, $\beta_{2}^{(2)}=1.8 \mathrm{ps}^{2} / \mathrm{km}$, and $\mathrm{FSR}_{2}=221.45 \mathrm{GHz}$. Accordingly, in the short $3 \times 3$ coupling region described by Eq. (1), the cross-coupling powers between the two microresonator transverse modes are orders of magnitude smaller than the corresponding self-coupling terms (similar to earlier reports [50]). The nonlinear coefficients are $\gamma_{1}=0.89(\mathrm{~m} \mathrm{~W})^{-1}$ and $\gamma_{2}=0.44(\mathrm{~m} \mathrm{~W})^{-1}$, and the ring length is $L=2 \pi \times 100 \mu \mathrm{m}$. The linear coupling between the two modes is $\kappa_{12}=22.7 \mathrm{~m}^{-1}$, calculated from the measurements in Fig. 1(d). The pump detuning is varied linearly in a dynamic manner, such that the final detuning of the main mode is $\delta_{0}^{(1)}=0.02 \mathrm{rad}$ after $750 \mathrm{~ns}$. After the field inside the cavity has stabilized and converged to a steady state, the results are analyzed.

The simulated comb envelope for various pump detunings and their corresponding simulated intracavity waveforms are shown in red in Fig. 2(d). The corresponding frequency lines of the measured comb spectra are also depicted in Fig. 2(d). The simulated waveforms reveal that in the forward pump tuning, with each step in the comb power, one additional low intensity oscillation appears at the center of the dark-pulse structure. The observed states correspond to different snaking branch solutions reported in the bifurcation analysis of switching waves [30]. In cavities with a single transverse mode, where there is no linear mode coupling $(\kappa=0)$, the snaking branch of solutions are disconnected and have discrete steps, as shown in the simulations of Fig. 3 (dashed red curve) [30]. However, as seen in Figs. 2(b) and 2(c) in our experiments we observe a continuous curve as the pump is tuned. The continuity is due to the linear coupling between the transverse modes, which merges the discrete steps into a continuous curve and enables gradual switching between dark-pulse comb states (dotted blue and solid green curves in Fig. 3), as opposed to the abrupt jumps observed in DKS [14]. In the time domain, this translates into continuous tuning of the shape and number (a)

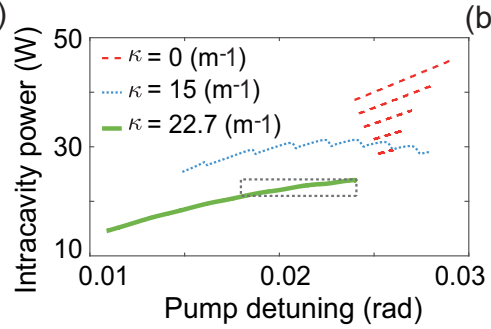

(b)

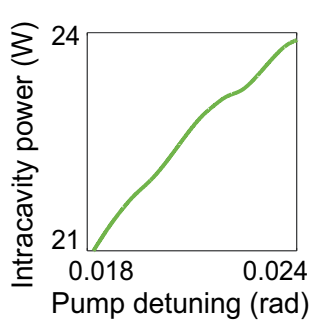

FIG. 3. (a) Simulation of average intracavity power for different linear coupling coefficients $\kappa$, as the pump is forward tuned. (b) Zoomed-in view of the dashed region in (a), clearly showing smooth steps similar to those observed in our experiments [Fig. 2(c)]. 
of the low-intensity oscillations in the dark pulse. Thus, as the pump is tuned across one step, the shape of the oscillations gradually changes until the switching point, where finally an additional oscillation appears. For higher $\kappa$, the shape of the oscillations vary slower and thus sharp changes are not observed in the pulse power (solid green curve in Fig. 3). This feature, which has been previously predicted [34], is observed here.

After accessing a comb state in the forward tuning, the switching transition can be reversed by tuning the pump backwards [yellow (light gray) curve in Fig. 2(b)]. The comb power drops and shows a smoothed steplike pattern similar to the forward tuning, but in the reverse direction. Thus, the low-intensity oscillations in the dark pulse vanish one by one, until what appears to be a single gray soliton state [54-56] is accessed [state F in Fig. 2(d)]. However, strictly speaking it is not a gray soliton in the sense of the dissipationless nonlinear Schrödinger equation, as the temporal phase is not an odd function of time due to the periodic boundary conditions of the cavity. Switching occurs over a broader detuning range in the backward pump tuning (state $\mathrm{C}$ to $\mathrm{F}$ ) compared to forward tuning (state A to $\mathrm{C}$ ), giving access to more comb states. The comb power in the forward and backward pump tuning shows a hysteresis behavior, similar to what has been observed for DKS in the anomalous dispersion regime [14].

We find an excellent agreement between the measured and simulated comb spectra, indicating that by just measuring the transmission spectrum and retrieving the parameters of the interacting modes, one can predict the comb dynamics starting from a CW pump by using two linearly coupled equations. Moreover, the excellent agreement between the experimental and simulated comb spectra confirms that linear mode coupling is the dominant cause leading to the generation of the dark-pulse comb. The discrepancy between simulations and experiments for longer wavelengths in Fig. 2(d) might be due to a second mode coupling around $1590 \mathrm{~nm}$. The measurements display an asymmetry in the comb spectra, which had also been observed in previously reported darkpulse Kerr combs [36]. Our simulations naturally capture this comb asymmetry even though the third-order dispersion has not been included, clearly indicating that the asymmetry is caused by the linear coupling between the two transverse modes.

\section{HOT-CAVITY SPECTROSCOPY OF DARK-PULSE KERR COMBS}

To get a better understanding of the dynamics of dark-pulse Kerr combs, we look into the system's response upon the dark-pulse formation. The formation of a dark pulse breaks the time invariance of the system, making it impossible to describe the resonator in terms of a linear transfer function. Instead, we measure the system's response with the aid of an external (probe) laser, as sketched in Fig. 4(a). A reddetuned probe laser with a fixed frequency, far detuned from the cavity resonances, is weakly modulated with an external dual-sideband electro-optic intensity modulator, driven by a tunable radio-frequency ( $\mathrm{RF}$ ) signal, allowing to retrieve the system's response as the pump laser is tuned into resonance [setup shown in Fig. 2(a)]. The benefit of using this scheme instead of modulating the pump itself is that it solves the ambiguity in the pump location with respect to the resonances of the coupled modes. One of the two generated probe sidebands is scanned across the cavity resonances of the two interacting modes, by sweeping the RF source from $10 \mathrm{MHz}$ to $24 \mathrm{GHz}$ in $10 \mathrm{MHz}$ steps. The sideband will be affected by the presence of resonances in the cavity and nonlinear distortions caused by the pump and the dark pulse. A vector network analyzer (VNA) measures the magnitude of the RF beat between the sideband and probe laser as the sideband is swept in frequency. This process is repeated as the pump laser is tuned across the hybridized cavity resonances [resonances of the two linearly coupled modes labeled as 1 and 2 in Fig. 4(a)] from the blue side in 81 steps. The recorded system's responses are displayed in Fig. $4(\mathrm{~b})$. The parameter $\Delta \mathrm{f}_{\text {pump }}$ determines the location of the pump laser, while $\Delta \mathrm{f}_{\mathrm{VNA}}$ is the detuning of the sideband, both measured relative to the probe laser. As the laser is tuned closer to the resonances, $\Delta \mathrm{f}_{\text {pump }}$ decreases and varies from $23.94 \mathrm{GHz}$ down to $1.053 \mathrm{GHz}$. The two lasers are not locked to each other, so the measurement of the system's response has a frequency resolution in the order of a few $\mathrm{MHz}$, given by the relative drift between the probe and pump lasers. Since the pump frequency varies in every step, the beat note between the pump and sideband is used to find the location of the pump.

At the initial stages prior to the formation of the dark-pulse Kerr comb, the system's response is affected by thermal dynamics. Consequently, the hybridized resonances are strongly red shifted, which decreases their $\Delta \mathrm{f}_{\mathrm{VNA}}$ [states I and II in Figs. 4(b), 4(d)]. By further tuning the pump, a mode-locked dark-pulse comb emerges suddenly (state III) as soon as the pump crosses the first hybridized resonance, labeled "2" (see Supplemental Material [57]). The generation of a dark-pulse Kerr comb is associated with the emergence of a third resonance [labeled " 3 " in Fig. 4(a) and visible in states III and IV in Figs. 4(b), 4(d)]. This feature has a striking similarity to observations made for DKS [14]. In this stage, the pump frequency is effectively red detuned with respect to this resonance. Once the pump crosses the hybridized resonance 2, this resonance moves towards higher frequencies. Our simulations, explained in the following [see Fig. 4(e)], confirm that the generated resonance 3 is the resonance located closer to the pump, while the resonance further away from the pump is the hybridized resonance 2 . Further moving the pump to the red side causes the laser to cross the two hybridized resonances, associated with a loss of the comb and a cooling down of the system [see Fig. 4(b)]. The system's response in this case is similar to that of the first stage, with two Lorentzian shape resonances associated with the hybridized modes [state I in Fig. 4(d)].

We explain the appearance of the third resonance as follows. Dark pulses are intermediate solutions between the upper and lower CW steady-state solutions of the Lugiato-Lefever equation [5,58-60]. Given the intensity dependence of the Kerr effect, the high-intensity CW background and the low-intensity oscillations in the dark pulse induce different nonlinear Kerr phase shifts on the cavity resonances, but most notably on the main mode. In particular, the high-power level shifts the cavity resonance to the red side of the pump, while the low-power level induces a smaller 
(a)
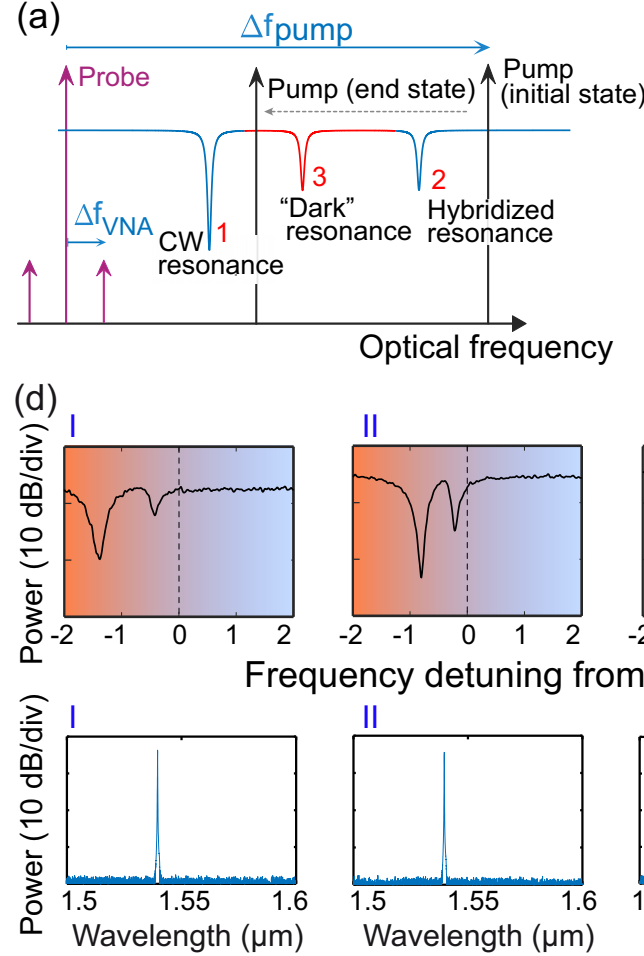

(b)

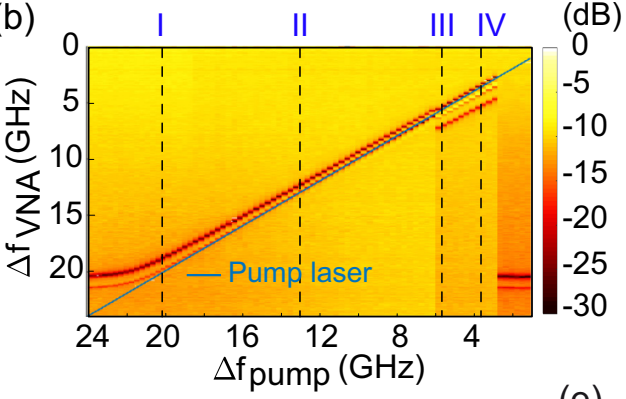

(c)

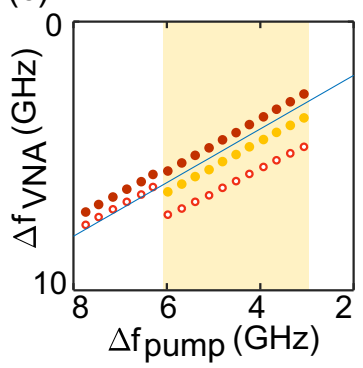

III

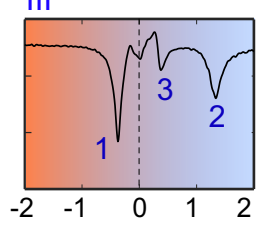

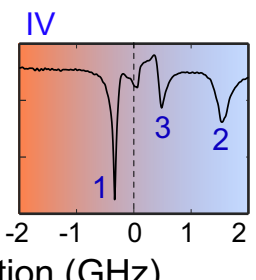

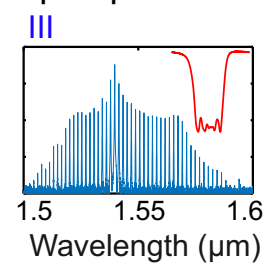

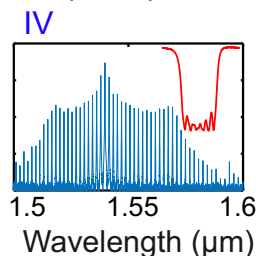

(e)
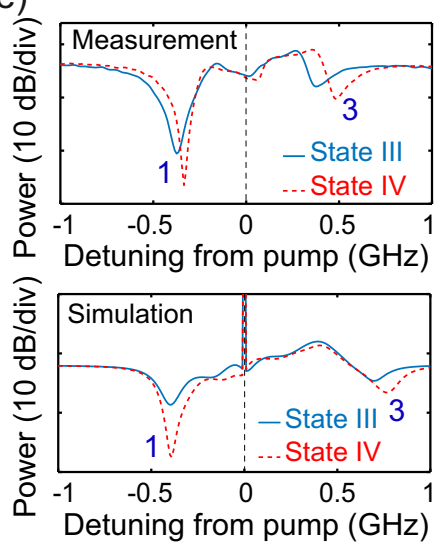

FIG. 4. (a) Schematic diagram of the method used for the measurement of the system response. The pump approaches the hybridized resonances 1 and 2 from the blue side. Once the pump crosses resonance 2, a dark-pulse comb is generated and another resonance, labeled 3 , emerges. (b) System response as the pump is tuned into resonance. (c) Zoomed-in view of (b), where the existence of dark pulses is highlighted. The appearance of an extra resonance can be clearly observed in this regime. (d) VNA traces and corresponding measured comb spectra and simulated time-domain waveforms (inset) for various pump detunings indicated by dashed lines in (b). Note that the origin of the frequency axis here is the pump laser, providing a direct indication of the location of the resonances and effective pump-laser detuning. (e) Measured and simulated VNA traces associated with the main mode for pump detunings III and IV in (b). The experimental traces are the zoomed-in view of states III and IV in (d).

shift on the cavity resonance and creates a resonance on the blue side of the pump. This behavior is analogous to the appearance of the soliton resonance in the system response of anomalous dispersion microresonators [14]. In dark-pulse Kerr combs, a subtle yet important difference is that the $\mathrm{CW}$ background corresponds to the high-power level, so its Kerr shifted resonance appears on the red side of the pump. Meanwhile, the low-intensity oscillations at the center of the pulse, which are associated with the generation of the dark pulse, generate another resonance on the blue side of the pump labeled as "dark resonance" [Fig. 4(a)]. Hence, in contrast to DKS, where the CW background is weak and the soliton has a high-power level, for dark-pulse Kerr combs the pump laser remains on the effectively blue-detuned side with respect to the CW background resonance [labeled " 1 " in Fig. 4(a)]. The observations made here are consistent with previous studies based on modulation of the resonator using a microheater [29]. The emergence of an extra resonance is a unique property of stationary solitonic states in Kerr microresonators that had not been previously demonstrated for dark-pulse Kerr combs.

We support the explanation of the VNA response above with numerical simulations. For a complete numerical analysis, as explained in Sec. III, the comb generation can be simulated based on the linear coupling between the two transverse modes, while considering quantum noise as the initial intracavity field. The auxiliary mode and the linear coupling between the modes are essential, as they play an important role in initiating the comb generation. However, to simplify the simulations, if we assume that the comb has already been initiated, or in other words if instead of the quantum noise, a field is already circulating in the cavity, then it is no longer necessary to include the auxiliary mode and linear mode coupling in the comb generation simulations. Therefore, here, for simplicity, we assume only a single transverse mode in the cavity. Meanwhile, the initial intracavity field is considered as a square dark pulse, where the amplitude and phase of the top (bottom) of the pulse are equal to the upper branch (lower branch) steady-state values [29]. This is a reasonable assumption, given that the appearance of the additional resonance in the system's response arises from the two power levels (high-intensity CW background and low-intensity oscillations of the dark pulse) present in the intracavity waveform of the main mode and does not directly depend on the auxiliary mode nor the linear coupling between the modes. The considered ring parameters and pump power are similar to the main mode values mentioned in Sec. III. The pump is fixed in frequency and a weak $(-40 \mathrm{dBm})$ probe is swept across the resonance in $10 \mathrm{MHz}$ steps. In each step, after simulating the output spectrum of the microresonator, the power of the probe frequency component is calculated. The comparison between this power and the probe power at the input is the system's 
(a)

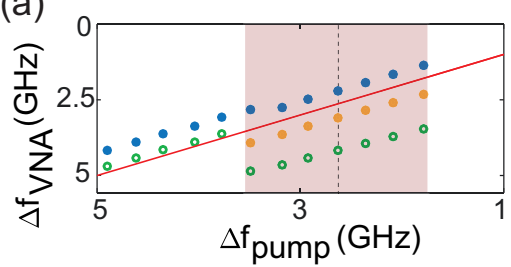

(b)

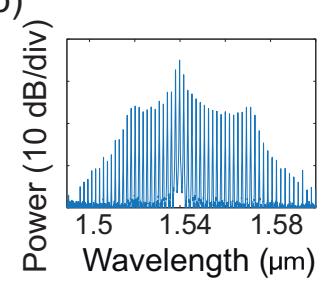

FIG. 5. Hot-cavity spectroscopy of a dark-pulse Kerr comb in a different silicon nitride microresonator with similar characteristics, in terms of dispersion, $Q$ and avoided mode crossing. (a) System response as the pump is tuned into resonance from the blue side. The appearance of a third resonance can be clearly observed in the dark-pulse regime (highlighted region). (b) Dark-pulse Kerr comb spectrum measured at the pump detuning indicated by a dashed line in (a).

response. It corresponds to the beat note between the sideband and the probe, measured with the VNA in the experiment. The considered cold-cavity detunings are $\delta_{0}^{(1)}=0.0242 \mathrm{rad}$ and $\delta_{0}^{(1)}=0.0248 \mathrm{rad}$, which correspond to the comb states III and IV in Fig. 4(d), respectively. The measured and simulated system responses of these two pump detunings are shown in Fig. 4(e). The appearance of an extra resonance is evident in the simulations too. Both measured and simulated results indicate that the depth of the $\mathrm{CW}$ resonance increases with the red tuning of the pump. Moreover, the frequency of the $\mathrm{CW}$ resonance remains almost fixed, while that of the dark resonance shifts with the pump detuning. This shift is related to changes in the lower level of the dark-pulse structure, which correspond to dark pulses with different number of oscillations [shown in the inset of Fig. 4(d)]. The power variations of the VNA traces around the pump frequency, observed in both experiments and simulations, are related to the nonlinear effects induced on the sideband; an aspect that has also been observed in other experiments using other microresonator platforms [61]. Note that the switching between dark-pulse comb states is not associated with sharp changes in the system's response. The reason is that switching in dark pulses changes the number of oscillations, which have a low intensity and do not introduce a significant energy change in the cavity.

To show the generality of our observations, we also measured the system's response in a second silicon nitride microresonator chip. The microresonator has nominally the

same dimensions as the chip used in our main experiments, except for the gap between the ring and the drop port. The measured results are shown in Fig. 5. The formation of a dark-pulse Kerr comb in this microresonator is also clearly accompanied by the emergence of an additional resonance.

\section{CONCLUSIONS}

The physics of dark-pulse Kerr comb generation and its switching dynamics are investigated, both experimentally and numerically. Deterministic switching between dark-pulse comb states is observed. Numerical simulations, which give rise to frequency combs that are in excellent agreement with the measured spectra, suggest that as the pump tuning varies, the number of low-intensity oscillations at the center of the corresponding dark pulses can either increase or decrease, one at a time. Moreover, we measure the system's response as the pump laser is tuned into resonance and discover that the formation of a dark-pulse Kerr comb is associated with the emergence of an extra resonance. This is due to the combination of nonlinearity and bistability in the cavity. The revealed multiresonance dynamics is a distinctive property of soliton states in Kerr cavities and confirms the switching behavior of the dark-pulse combs in a new way. Furthermore, by using an external probe to measure the system's response, we could disentangle the different resonances present in the system, clearly indicating that for dark-pulse states, the pump laser lies in the effectively blue-detuned region with respect to the $\mathrm{CW}$ background resonance, in sharp contrast to DKS in anomalous dispersion microresonators. These results shed light into the formation of Kerr combs in normal dispersion microresonators and pave the way for the generation of reproducible chip-scale comb sources with high-power conversion efficiency.

The measured raw data necessary to reproduce the plots in this work can be accessed at [62].

\section{ACKNOWLEDGMENTS}

This work was supported in part by the European Research Council (ERC CoG, Grant Agreement No. 771410 DarkComb), Swedish Research Council (VR), the National Science Foundation (NSF) (1809784-ECCS), DARPA (W31P40-13-1-001.8), and AFOSR (FA9550-15-1-0211).
[1] N. Akhmediev and A. Ankiewicz, Dissipative Solitons: From Optics to Biology and Medicine (Springer, Berlin, 2008).

[2] F. Leo, S. Coen, P. Kockaert, S.-P. Gorza, P. Emplit, and M. Haelterman, Temporal cavity solitons in one-dimensional Kerr media as bits in an all-optical buffer, Nature Photonics 4, 471 (2010).

[3] T. Herr, V. Brasch, J. D. Jost, C. Y. Wang, N. M. Kondratiev, M. L. Gorodetsky, and T. J. Kippenberg, Temporal solitons in optical microresonators, Nature Photonics 8, 145 (2013).

[4] T. J. Kippenberg, A. L. Gaeta, M. Lipson, and M. L. Gorodetsky, Dissipative Kerr solitons in optical microresonators, Science 361, eaan8083 (2018).
[5] C. Godey, I. V. Balakireva, A. Coillet, and Y. K. Chembo, Stability analysis of the spatiotemporal Lugiato-Lefever model for Kerr optical frequency combs in the anomalous and normal dispersion regimes, Phys. Rev. A 89, 063814 (2014).

[6] S. Coen and M. Erkintalo, Universal scaling laws of Kerr frequency combs, Opt. Lett. 38, 1790 (2013).

[7] A. B. Matsko, A. A. Savchenkov, and L. Maleki, On excitation of breather solitons in an optical microresonator, Opt. Lett. 37, 4856 (2012).

[8] C. Bao, J. A. Jaramillo-Villegas, Y. Xuan, D. E. Leaird, M. Qi, and A. M. Weiner, Observation of Fermi-Pasta-Ulam 
Recurrence Induced by Breather Solitons in an Optical Microresonator, Phys. Rev. Lett. 117, 163901 (2016).

[9] M. Yu, J. K. Jang, Y. Okawachi, A. G. Griffith, K. Luke, S. A. Miller, X. Ji, M. Lipson, and A. L. Gaeta, Breather soliton dynamics in microresonators, Nature Commun. 8, 14569 (2017).

[10] E. Lucas, M. Karpov, H. Guo, M. L. Gorodetsky, and T. J. Kippenberg, Breathing dissipative solitons in optical microresonators, Nature Commun. 8, 736 (2017).

[11] D. C. Cole, E. S. Lamb, P. Del'Haye, S. A. Diddams, and S. B. Papp, Soliton crystals in Kerr resonators, Nature Photonics 11, 671 (2017).

[12] W. Wang, Z. Lu, W. Zhang, S. T. Chu, B. E. Little, L. Wang, X. Xie, M. Liu, Q. Yang, L. Wang, J. Zhao, G. Wang, Q. Sun, Y. Liu, Y. Wang, and W. Zhao, Robust soliton crystals in a thermally controlled microresonator, Opt. Lett. 43, 2002 (2018).

[13] M. Karpov, M. H. P. Pfeiffer, H. Guo, W. Weng, J. Liu, and T. J. Kippenberg, Dynamics of soliton crystals in optical microresonators, Nature Phys. 15, 1071 (2019).

[14] H. Guo, M. Karpov, E. Lucas, A. Kordts, M. H. P. Pfeiffer, V. Brasch, G. Lihachev, V. E. Lobanov, M. L. Gorodetsky, and T. J. Kippenberg, Universal dynamics and deterministic switching of dissipative Kerr solitons in optical microresonators, Nature Phys. 13, 94 (2017).

[15] A. Pasquazi, M. Peccianti, L. Razzari, D. J. Moss, S. Coen, M. Erkintalo, Y. K. Chembo, T. Hansson, S. Wabnitz, P. Del'Haye, X. Xue, A. M. Weiner, and R. Morandotti, Micro-combs: A novel generation of optical sources, Phys. Rep. 729, 1 (2018).

[16] P. Marin-Palomo, J. N. Kemal, M. Karpov, A. Kordts, J. Pfeifle, M. H. P. Pfeiffer, P. Trocha, S. Wolf, V. Brasch, M. H. Anderson, R. Rosenberger, K. Vijayan, W. Freude, T. J. Kippenberg, and C. Koos, Microresonator-based solitons for massively parallel coherent optical communications, Nature (London) 546, 274 (2017).

[17] M.-G. Suh, Q.-F. Yang, K. Y. Yang, X. Yi, and K. J. Vahala, Microresonator soliton dual-comb spectroscopy, Science 354, 600 (2016).

[18] W. Liang, D. Eliyahu, V. S. Ilchenko, A. A. Savchenkov, A. B. Matsko, D. Seidel, and L. Maleki, High spectral purity Kerr frequency comb radio frequency photonic oscillator, Nature Commun. 6, 7957 (2015).

[19] P. Trocha, M. Karpov, D. Ganin, M. H. P. Pfeiffer, A. Kordts, S. Wolf, J. Krockenberger, P. Marin-Palomo, C. Weimann, S. Randel, W. Freude, T. J. Kippenberg, and C. Koos, Ultrafast optical ranging using microresonator soliton frequency combs, Science 359, 887 (2018).

[20] M.-G. Suh and K. J. Vahala, Soliton microcomb range measurement, Science 359, 884 (2018).

[21] Q.-F. Yang, B. Shen, H. Wang, M. Tran, Z. Zhang, K. Y. Yang, L. Wu, C. Bao, J. Bowers, A. Yariv, and K. Vahala, Vernier spectrometer using counterpropagating soliton microcombs, Science 363, 965 (2019).

[22] D. T. Spencer, T. Drake, T. C. Briles, J. Stone, L. C. Sinclair, C. Fredrick, Q. Li, D. Westly, B. R. Ilic, A. Bluestone, N. Volet, T. Komljenovic, L. Chang, S. H. Lee, D. Y. Oh, M.-G. Suh, K. Y. Yang, M. H. P. Pfeiffer, T. J. Kippenberg, E. Norberg, L. Theogarajan, K. Vahala, N. R. Newbury, K. Srinivasan, J. E. Bowers, S. A. Diddams, and S. B. Papp, An optical-frequency synthesizer using integrated photonics, Nature (London) 557, 81 (2018).
[23] E. Obrzud, M. Rainer, A. Harutyunyan, M. H. Anderson, J. Liu, M. Geiselmann, B. Chazelas, S. Kundermann, S. Lecomte, M. Cecconi, A. Ghedina, E. Molinari, F. Pepe, F. Wildi, F. Bouchy, T. J. Kippenberg, and T. Herr, A microphotonic astrocomb, Nature Photonics 13, 31 (2019).

[24] M.-G. Suh, X. Yi, Y.-H. Lai, S. Leifer, I. S. Grudinin, G. Vasisht, E. C. Martin, M. P. Fitzgerald, G. Doppmann, J. Wang, D. Mawet, S. B. Papp, S. A. Diddams, C. Beichman, and K. Vahala, Searching for exoplanets using a microresonator astrocomb, Nature Photonics 13, 25 (2019).

[25] T. E. Drake, T. C. Briles, J. R. Stone, D. T. Spencer, D. R. Carlson, D. D. Hickstein, Q. Li, D. Westly, K. Srinivasan, S. A. Diddams, and S. B. Papp, Terahertz-Rate KerrMicroresonator Optical Clockwork, Phys. Rev. X 9, 031023 (2019).

[26] X. Xue, X. Zheng, B. Zhou, and A. M. Weiner, Microresonator frequency combs for integrated microwave photonics, IEEE Photonics Technol. Lett. 30, 1814 (2018).

[27] Note that we use the standard terminology in nonlinear [63] and ultrafast optics [64], where anomalous dispersion refers to a negative group velocity dispersion coefficient, whereby the blue components in the microresonator travel faster than the red ones, leading to an increasing FSR with optical frequency.

[28] S.-W. Huang, H. Zhou, J. Yang, J. F. McMillan, A. Matsko, M. Yu, D.-L. Kwong, L. Maleki, and C. W. Wong, ModeLocked Ultrashort Pulse Generation From On-Chip Normal Dispersion Microresonators, Phys. Rev. Lett. 114, 053901 (2015).

[29] X. Xue, Y. Xuan, Y. Liu, P.-H. Wang, S. Chen, J. Wang, D. E. Leaird, M. Qi, and A. M. Weiner, Mode-locked dark pulse Kerr combs in normal-dispersion microresonators, Nature Photonics 9, 594 (2015).

[30] P. Parra-Rivas, D. Gomila, E. Knobloch, S. Coen, and L. Gelens, Origin and stability of dark pulse Kerr combs in normal dispersion resonators, Opt. Lett. 41, 2402 (2016).

[31] C. Bao, Y. Xuan, C. Wang, A. Fülöp, D. E. Leaird, V. TorresCompany, M. Qi, and A. M. Weiner, Observation of Breathing Dark Pulses in Normal Dispersion Optical Microresonators, Phys. Rev. Lett. 121, 257401 (2018).

[32] J. Peng, S. Boscolo, Z. Zhao, and H. Zeng, Breathing dissipative solitons in mode-locked fiber lasers, Sci. Adv. 5, eaax1110 (2019).

[33] B. Varlot, S. Wabnitz, J. Fatome, G. Millot, and C. Finot, Experimental generation of optical flaticon pulses, Opt. Lett. 38, 3899 (2013).

[34] V. Lobanov, G. Lihachev, T. J. Kippenberg, and M. Gorodetsky, Frequency combs and platicons in optical microresonators with normal GVD, Opt. Express 23, 7713 (2015).

[35] X. Xue, P.-H. Wang, Y. Xuan, M. Qi, and A. M. Weiner, Microresonator Kerr frequency combs with high conversion efficiency, Laser Photon. Rev. 11, 1600276 (2017).

[36] A. Fülöp, M. Mazur, A. Lorences-Riesgo, Ó. B. Helgason, P.-H. Wang, Y. Xuan, D. E. Leaird, M. Qi, P. A. Andrekson, A. M. Weiner, and V. Torres-Company, High-order coherent communications using mode-locked dark-pulse Kerr combs from microresonators, Nature Commun. 9, 1598 (2018).

[37] Ó. B. Helgason, A. Fülöp, J. Schröder, P. A. Andrekson, A. M. Weiner, and V. Torres-Company, Superchannel engineering of microcombs for optical communications, J. Opt. Soc. Am. B 36, 2013 (2019). 
[38] S. Burger, K. Bongs, S. Dettmer, W. Ertmer, K. Sengstock, A. Sanpera, G. V. Shlyapnikov, and M. Lewenstein, Dark Solitons in Bose-Einstein Condensates, Phys. Rev. Lett. 83, 5198 (1999).

[39] A. Chabchoub, O. Kimmoun, H. Branger, N. Hoffmann, D. Proment, M. Onorato, and N. Akhmediev, Experimental Observation of Dark Solitons on the Surface of Water, Phys. Rev. Lett. 110, 124101 (2013).

[40] P.-H. Wang, Y. Xuan, L. Fan, L. T. Varghese, J. Wang, Y. Liu, X. Xue, D. E. Leaird, M. Qi, and A. M. Weiner, Drop-port study of microresonator frequency combs: power transfer, spectra and time-domain characterization, Opt. Express 21, 22441 (2013).

[41] D. Marcuse, Theory of Dielectric Optical Waveguides (Academic Press, New York, 1991).

[42] T. Klaassen, J. de Jong, M. van Exter, and J. P. Woerdman, Transverse mode coupling in an optical resonator, Opt. Lett. 30, 1959 (2005).

[43] S. Gröblacher, K. Hammerer, M. R. Vanner, and M. Aspelmeyer, Observation of strong coupling between a micromechanical resonator and an optical cavity field, Nature (London) 460, 724 (2009).

[44] Y. Liu, Y. Xuan, X. Xue, P.-H. Wang, S. Chen, A. J. Metcalf, J. Wang, D. E. Leaird, M. Qi, and A. M. Weiner, Investigation of mode coupling in normal-dispersion silicon nitride microresonators for Kerr frequency comb generation, Optica 1, 137 (2014).

[45] T. Carmon, L. Yang, and K. J. Vahala, Dynamical thermal behavior and thermal self-stability of microcavities, Opt. Express 12, 4742 (2004).

[46] K. Ikeda, Multiple-valued stationary state and its instability of the transmitted light by a ring cavity system, Opt. Commun. 30, 257 (1979).

[47] V. Torres-Company, D. Castelló-Lurbe, and E. Silvestre, Comparative analysis of spectral coherence in microresonator frequency combs, Opt. Express 22, 4678 (2014).

[48] T. Hansson and S. Wabnitz, Frequency comb generation beyond the lugiato-lefever equation: multi-stability and super cavity solitons, J. Opt. Soc. Am. B 32, 1259 (2015).

[49] W.-P. Huang, Coupled-mode theory for optical waveguides: an overview, J. Opt. Soc. Am. A 11, 963 (1994).

[50] R. Stoffer, K. R. Hiremath, M. Hammer, L. Prkna, and J. Čtyroký, Cylindrical integrated optical microresonators: Modeling by 3-d vectorial coupled mode theory, Opt. Commun. 256, 46 (2005).
[51] F. Poletti and P. Horak, Description of ultrashort pulse propagation in multimode optical fibers, J. Opt. Soc. Am. B 25, 1645 (2008).

[52] A. Mafi, Pulse propagation in a short nonlinear graded-index multimode optical fiber, J. Lightwave Technol. 30, 2803 (2012).

[53] G. D'Aguanno and C. R. Menyuk, Nonlinear mode coupling in whispering-gallery-mode resonators, Phys. Rev. A 93, 043820 (2016).

[54] W. J. Tomlinson, R. J. Hawkins, A. M. Weiner, J. P. Heritage, and R. N. Thurston, Dark optical solitons with finite-width background pulses, J. Opt. Soc. Am. B 6, 329 (1989).

[55] A. M. Weiner, J. P. Heritage, R. J. Hawkins, R. N. Thurston, E. M. Kirschner, D. E. Leaird, and W. J. Tomlinson, Experimental Observation of the Fundamental Dark Soliton in Optical Fibers, Phys. Rev. Lett. 61, 2445 (1988).

[56] Y. S. Kivshar and B. Luther-Davies, Dark optical solitons: physics and applications, Phys. Rep. 298, 81 (1998).

[57] See Supplemental Material at http://link.aps.org/supplemental/ 10.1103/PhysRevA.103.013513 for a video illustrating the detuning dynamics and formation of the additional resonance.

[58] L. A. Lugiato and R. Lefever, Spatial Dissipative Structures in Passive Optical Systems, Phys. Rev. Lett. 58, 2209 (1987).

[59] S. Coen, H. G. Randle, T. Sylvestre, and M. Erkintalo, Modeling of octave-spanning Kerr frequency combs using a generalized mean-field lugiato-lefever model, Opt. Lett. 38, 37 (2013).

[60] M. Haelterman, S. Trillo, and S. Wabnitz, Dissipative modulation instability in a nonlinear dispersive ring cavity, Opt. Commun. 91, 401 (1992).

[61] G. N. Ghalanos, J. M. Silver, L. D. Bino, N. P. Moroney, M. T. M. Woodley, A. Svela, S. Zhang, and P. Del'Haye, Direct measurement of cross-phase modulation in microresonators, in 2019 Conference on Lasers and Electro-Optics Europe and European Quantum Electronics Conference (IEEE, 2019), p. 1.

[62] E. Nazemosadat, A. Fülöp, Ó. B. Helgason, P.-H. Wang, Y. Xuan, D. E. Leaird, M. Qi, E. Silvestre, A. M. Weiner, and V. Torres-Company, dataset for "Switching dynamics of darkpulse Kerr comb states in optical microresonators", 2020, doi: 10.5281/zenodo.4284030.

[63] G. P. Agrawal, Nonlinear Fiber Optics, 4th ed. (Academic Press, San Diego, 2007).

[64] A. Weiner, Ultrafast Optics (John Wiley \& Sons, New York, 2011). 